\title{
Decubitus Ulcers In Patients Undergoing Vascular Operations Do Not Influence Mortality But Affect Resource Utilization
}

\author{
J. Hunter Mehaffey, MD'1, Amani D Politano, MD, MS ${ }^{1}$, Castigliano M Bhamidipati, DO, PhD, \\ MSc $^{2}$, Margaret C Tracci, MD, JD ${ }^{1}$, Kenneth J Cherry, MD ${ }^{1}$, John A Kern, MD ${ }^{1}$, Irving L \\ Kron, $\mathbf{M D}^{1}$, and Gilbert R Upchurch Jr., MD ${ }^{1}$ \\ ${ }^{1}$ Division of Vascular and Endovascular Surgery, Department of Surgery, University of Virginia \\ Health System, Charlottesville, Virginia \\ ${ }^{2}$ Division of Cardiothoracic Surgery, Department of Surgery, University of Washington, Seattle \\ Washington
}

\begin{abstract}
Background-While it is anticipated that decubitus ulcers (DU) are detrimental to outcomes following vascular operations, the contemporary influence of perioperative DU in vascular surgery remains unknown.
\end{abstract}

Methods-Using the National Impatient Survey (NIS), all adult patients who underwent vascular surgery were selected. Patients were stratified by the presence or absence (non-DU) of decubitus ulcer. Case-mix adjusted hierarchical mixed-models examined in-hospital mortality, the occurrence of any complication, and discharge disposition.

Results-A total of 538,808 cases were analyzed. DU was most prevalent among Caucasian, male, Medicare beneficiaries ( $\mathrm{p}<0.001)$. DU patients also underwent more non-elective vascular surgery $(\mathrm{p}<0.001)$. Wound, infectious, and procedural complications were more common in patients with DU ( $\mathrm{p}<0.001)$. Failure to rescue, defined as mortality following any complication, was more than doubled in DU (non-DU: $1.5 \%$, DU: $3.2 \%$, p<0.001). Similarly, unadjusted mortality was also doubled in patients undergoing vascular surgery with DU (non-DU: 3\%, DU: $6 \%, \mathrm{p}<0.001)$. Following risk adjustment among all patients, neither the presence of a DU nor specific ulcer staging increased the adjusted odds of death. Having a DU increased the adjusted odds of discharge to an intermediate care facility (OR 2.9, $\mathrm{p}<0.001$ ). These patients also had 1.6 times the total charges compared to their non-DU cohort (non-DU: $\$ 49,460 \pm 281$ vs. DU:

$\$ 81,149 \pm 5855, \mathrm{p}<0.001)$.

Conclusions-Contrary to common perception, perioperative decubitus ulcer does not adversely affect mortality following vascular surgery in patients proceeding to surgical intervention. Patients

Correspondence: Gilbert R Upchurch Jr., MD, Professor of Surgery, Division of Vascular and Endovascular Surgery, Department of Surgery, University of Virginia School of Medicine, Box 800679, Charlottesville, Virginia 22908, Phone: (434) 243-6333, Fax: (434) 243-9941, gru6n@virginia.edu.

Publisher's Disclaimer: This is a PDF file of an unedited manuscript that has been accepted for publication. As a service to our customers we are providing this early version of the manuscript. The manuscript will undergo copyediting, typesetting, and review of the resulting proof before it is published in its final citable form. Please note that during the production process errors may be discovered which could affect the content, and all legal disclaimers that apply to the journal pertain. 
with decubitus ulcers are, however, at higher risk for complications and incur sizeable additional charges.

\section{Graphical abstract}

Using the NIS we evaluated the impact of DU on outcomes and resource utilization in vascular surgery. DU does not independently predict mortality but incurs higher risk for complications and a disproportionate increase in resource utilization.

\section{Introduction}

Decubitus (pressure) ulcers (DU) are a significant complication in many patient populations, including those who are elderly, frail, subject to prolonged bed rest, or nutritionally deficient, or in patients with spinal cord injuries (1-3). Additional contributing factors for DU include incontinence and altered consciousness $(4,5)$. Specifically in a general surgical population, additional risk factors for development of DU during hospitalization include low body mass index, use of vasopressors, multiple surgical interventions, and total operating time (6). In surgical intensive care units (ICU), the incidence of pressure ulcer development ranges between 3-9\%, with independent risk factors including age, elevated creatinine, diabetes, and spinal cord injury $(5,7)$.

The sequelae of developing DU include pain, infection, prolonged hospitalization, and increased risk of death $(2,8,9)$. Healthcare costs both during the hospitalization and for post- hospital care, such as nursing facility admissions or home health wound care, are also significantly increased in patients with DU $(1,2,10)$. DU represents a longitudinal disease process that can affect patients before, during and after hospital admission. Flattau and colleagues identified specific predictors associated with 90 and 180-day mortality in hospitalized patients with pressure ulcers, including liver disease and hypoalbuminemia (11). Other studies have demonstrated the long-term poor prognosis associated with DU due to comorbid disease $(1,4)$.

Vascular surgery patients often require several procedures or interventions in a single hospitalization, or may be relegated to mandatory bed rest during their stay (12).

Additionally, the predisposing conditions that lead to surgery in this population often indicate a globally compromised vascular supply that may increase risk of developing ulcerations. However, there are few studies directly addressing DU in the vascular surgery population, and none that assess the impact of DU in vascular patients with regard to postoperative outcomes and resource utilization $(7,13,14)$. The purpose of the present study is to determine whether the presence of DU increases mortality in patients undergoing major vascular procedures, and to assess the impact of procedure type, DU, and comorbid conditions on outcomes, including length of hospitalization, mortality, discharge disposition, and cost. 


\section{Methods}

\section{Data Sources}

Data was abstracted from the 2009 Nationwide Inpatient Sample (NIS). The NIS is the largest Healthcare Cost and Utilization Project (HCUP) all-payer inpatient database, sponsored by the Agency for Healthcare Research and Quality (AHRQ). The NIS contains data from more than 8 million hospital discharges annually from 1,050 hospitals located in 44 States, representing 95\% of all US nonfederal hospital discharges (www.hcupus.ahrq.gov/nisoverview). The AHRQ has developed appropriately scaled discharge weights to generate national estimates of hospitalizations from the NIS (http:// www.hcupus.ahrq.gov). These weights help compare hospitalization rates across years despite the varying number of states participating each year. The HCUP validates the NIS for biases by comparing it to other population-based datasets (http://www.hcup-us.ahrq.gov/ reports/methods.jsp). In this analysis when more than $2 \%$ of the variables for a particular record had data missing at random, we excluded the record from computations. No imputations were performed, and datasets were reviewed for any systematically missing values and accordingly excluded from evaluation. Data reporting meets the NIS data-use agreement as established by HCUP. The NIS databases contain de-identified administrative level data, and were not considered human subjects research; hence were exempted from review by the University of Virginia's Human Investigation Committee.

\section{Patient Selection}

Patients were selected based upon whether they underwent open or endovascular carotid repair, open abdominal aortic aneurysm repair, femoral artery to distal vessel revascularization, endovascular peripheral arterial stenting or above and/or below knee amputation using the International Statistical Classification of Diseases and Related Health Problems 9 Clinical Modification (ICD9-CM) codes. All fifteen procedure codes (PR1PR15) were queried to identify patients for having undergone operative procedures based on the following ICD9-CM codes: open carotid endarterectomy - 38.02, 38.12, 38.32, 38.42, 39.8 ; carotid artery stenting $-39.72,39.74,0.61,0.63$; open abdominal aneurysmectomy $38.04,38.06,38.14,38.16,38.34,38.36,38.44,38.46,39.24,39.25$, 39.26; endovascular abdominal stenting $-39.71,39.73$; open femoral artery to distal vessel bypass -38.08 , $38.18,38.38,38.48,39.29$; femoral artery to distal vessel endovascular revascularization 39.79; endovascular peripheral arterial stenting - 39.5; and above/below knee amputation $84.1,84.13,84.14,84.15,84.16,84.17,84.18,84.3,84.91$. Only patients over 18 years of age were selected. Cases with multiple procedural codes were assigned to groups based on the first procedure code to avoid the possibility of double counting any patient record. Patients with ruptured aneurysms were excluded. Records were selected only once per any given group (based on surgical procedure), and examined with the intent to perform a comprehensive analysis of the null hypothesis.

Patient risk factors were assessed using 30 different AHRQ comorbidities developed by Elixhauser. The Elixhauser comorbidities have been shown to provide effective adjustments for mortality risk among surgical populations, and have been shown to be superior to the Charlson/Deyo weighted score (15). 


\section{Decubitus Ulcers and Body Mass Index}

Based on ICD9-CM diagnosis codes, the selected patients from the above query were then identified as either having a decubitus ulcer in their hospital record or not. All 15 diagnosis codes (DX1-DX15) were queried to identify patients having a decubitus ulcer defined by the following ICD9-CM codes: 707, 707.01, 707.02, 707.03, 707.04, 707.05, 707.06, 707.07, 707.09, 707.2, 707.21, 707.22, 707.23, 707.24, 707.25. Additionally, based on ICD9-CM diagnosis codes, patients were also identified by the categorized body mass index (BMI) documented in their record. Several unadjusted and adjusted analyses were calculated to account for the influence of BMI on the presence or absence of a decubitus ulcer.

\section{Outcomes of Interest}

In-hospital mortality, complications and discharge disposition following major vascular surgery were our outcomes of interest. Complications were identified and limited to the hospital admission recorded ICD-9-CM codes. Because the NIS contains inpatient data only, complications occurring after hospital discharge cannot be evaluated. Several ICD-9-CM codes were adapted based on previously described work and were used to identify and aggregate complications into several categories including stroke, mechanical wound healing, postoperative infection, renal, pulmonary, gastrointestinal, cardiovascular, systemic, and procedural (16).

\section{Statistical Analysis}

The strength of the association between variables was examined using the appropriate hypothesis test. The significance of the differences between unadjusted proportions for categorical variables was evaluated by the Pearson $\chi^{2}$ test. Differences between mean values of unadjusted continuous variables were assessed using single factor analysis of variance models.

Unadjusted risk of complications, failure to rescue defined as the occurrence of mortality following any complication, discharge disposition, and in-hospital mortality across groups was calculated. Adjusted models using covariates as described below were included to calculate odds ratios for the response variable. Hierarchical multivariable regression models were developed to calculate the adjusted odds of an event occurring, by controlling for differences in case-mix, hospitalization, and administrative characteristics. The reference variables were selected based on clinical observation. Covariable selections for our models were made a priori based upon established literature(16-19). The models' predictive capacity to discriminate the response variable was measured using the area under the receiver operator characteristic curve (AUC). After excluding variables with missing values, more than $97 \%$ of the records were included in these analyses.

Model development may be error prone, and albeit regression modeling discriminates across parameters and identifies independent predictors associated with the response variable, accepting that the model is right (model validation) is equally important. Thus, we created randomly resampled equal split-groups from the original dataset and used the derivation dataset to develop models and the confirmatory dataset for validation. These two sets of models had minimal decrement (did not change by greater than 10\%). Following the 
assessment for robustness of models and stability of results, adjusted odds ratios (AOR) are presented for each covariate along with their $95 \%$ confidence interval (CI). All data were analyzed using IBM® SPSS ${ }^{\circledR}$ Complex Samples 20 (SPSS Inc., An IBM Co., Armonk, NY).

\section{Results}

\section{Demographics, comorbidities, and outcomes}

A total of 538,808 patients were examined, of who nearly 16,000 had DU. Comparison by procedure performed, demographics, and comorbidities is presented (Table 1). DU was more prevalent in Caucasians, males, and Medicare beneficiaries. Comorbid conditions that were more common in patients with DU included diabetes with chronic complications, neurologic disorders, obesity, paralysis, peripheral vascular disease, and renal failure. By admission characteristics, those with DU were more often admitted non-electively, on a weekend day, or as transfers from another hospital or health care facility (Table 2).

Unadjusted mortality was 2.3 -fold higher in patients with DU $(2.7 \%$ versus $6.3 \%, \mathrm{p}<0.001$, Table 3). Wound, infectious, systemic, and procedural complications were more frequent in this cohort as well. The prevalence of having two or more complications was 1.5 times higher in patients with DU. Additionally; patients with DU had longer mean lengths of stay by nearly 10 days. Failure to rescue, defined as mortality following any complication, was also higher in these patients.

\section{Adjusted risk of mortality}

Independent predictors of mortality among patients with DU included, by descending relative contribution, paralysis, electrolyte disorder, liver disease, non-elective admission, congestive heart failure, renal failure, and increasing age (Table 4). Weekend admission was associated with a 1.16-fold increase in risk of mortality (95\% confidence interval 1.02-1.31, $\mathrm{p}=0.021$ ). Conversely, diabetes (with and without chronic complications), neurologic disorders, and hypertension independently reduced the adjusted odds of death. Notably, DU did not independently increase the adjusted odds of mortality. A similar analysis assessing the impact of procedure performed on mortality demonstrated that carotid artery stent placement was associated with an increase in the adjusted odds of mortality, while endovascular peripheral artery stent placement was associated with a reduced risk (Table 5).

\section{Adjusted risk of developing any complication}

The adjusted odds of developing any complication during hospitalization for patients with DU were increased by the presence of chronic pulmonary disease, paralysis, congestive heart failure, electrolyte disorder, and non-elective admission, female gender, and increasing age. Diabetes (with and without chronic complications), renal failure, valvular heart disease, hypertension, collagen vascular disease, and liver disease reduced the adjusted odds of developing any complication (Table 4). Both open and endovascular abdominal aneurysm repair increased the adjusted risk of developing any complication, as did femoral-distal arterial stent placement to a lesser extent (Table 5). 


\section{Discharge disposition}

Patient disposition was significantly affected by the presence of DU (Table 6). For patients with DU, the independent likelihood of discharge to a skilled nursing facility is increased three-fold. In patients with DU who are admitted from a non-health care center for an elective surgery, the independent likelihood of discharge to a skilled nursing facility was increased 12 -fold.

\section{Total hospital charges}

Assessment of total hospital charges revealed significantly higher charges associated with presence of DU, at $\$ 128,997 \pm 1,222$ compared to $\$ 78,454 \pm 139$ for patients without DU. Component charges were not available to identify the highest cost aspects of care. When assessed by type of procedure performed, patients with DU accrued higher total hospital charges for every vascular procedure performed.

\section{Discussion}

Patients with decubitus ulcers are at risk for poorer outcomes and increased costs associated with hospitalizations $(1,4,20)$. However, this has not been assessed in the vascular surgery patient population. Many procedures these patients undergo may limit mobility either as a result of the primary procedure (e.g. amputation) or during recovery (e.g. tPA lysis), which can precipitate or worsen ulcers. Additionally, the comorbid conditions prevalent in this cohort increase the risk of complications and mortality $(2,11)$. To this end, the current study is the first to our knowledge that examines this association, and found that there was no association between the presence of ulcers and in hospital mortality, although resource utilization is greater.

While these data confirm our presupposition that patients undergoing vascular surgery with DU have nearly twice the mortality rate as patients without ulcers, when adjusted for several other patient factors, DU was no longer a significant predictor of mortality in this national cohort proceeding with surgical intervetion. Additionally, DU was not associated with an increased risk of any complications after adjustment. Rather DU serves as a marker of increased comorbid disease, which results in high morbidity and mortality in these chronically ill patients, but does not independently increase the risk of death. Vascular surgery patients likely have a higher rate of DU than other hospitalized patients given systemic cardiovascular disease with associated perfusion deficits. As a result, DU is an important comorbidity that vascular surgeons need to consider prior to undertaking semielective operations.

Several important risk factors were seen in these patients including comorbid disease as well as social and economical elements. Patients with DU were significantly more likely to have Medicare or Medicaid as the primary payor with half the rate of private insurance. Furthermore when assessing admission source DU patients were almost three times more likely to be transferred from another acute care hospital or other health care facility. These social factors have been previously demonstrated to have a major impact on outcomes.(17, 19) 
While mortality is not increased in these patients, the length of hospitalization and the rate of discharge to other health care facilities is higher, which in turn leads to much higher healthcare utilization in this population. These data demonstrate average cost increase of $\$ 50,000$ between patients with and without DU admitted for vascular surgery procedures. These differences were driven by significantly higher complications rates in all categories including wound infectious, renal, gastrointestinal, procedural, cardiovascular, neurologic and pulmonary. Additionally, patients with DU had higher total number of complications and almost three times longer hospital length of stay. Finally, patients with DU were over three times as likely to discharge to another healthcare facility resulting in significantly higher healthcare resource utilization.

Importantly, these data demonstrate that non-elective procedures should not be delayed or denied based on concerns for increased mortality. However, this study suggests elective procedures, which can be delayed, should be in order to optimize patients preoperatively. Realizing the impact that the presence of DU has in patients with regards to mortality, complications, and cost can aid vascular surgeons in preoperative planning and optimization. While many admissions in the present study were emergent, and therefore may not allow adequate time for preoperative measures, awareness of risk is critical in perioperative planning and patient counseling.

The limitations of this study include the retrospective nature of the study resulting in association, but an inability to establish causality. The NIS is a large administrative database providing a large sample size with limited clinical granularity and no details on treatment intention. Additionally, we are unable to ascertain if ulcers are present pre- or postoperatively, which may represent two distinct populations of patients. Finally, this is a 30,000 foot view and evaluates a variety of academic and community settings of various sizes and may not be directly translatable to individual centers.

\section{Conclusion}

The present data dispel the myth that patients with decubitus ulcers suffer higher mortality as a consequence of undergoing vascular operations. This suggests that the presence of an ulcer should not preclude vascular operations, as there is no detectable relationship with death in patients who proceed to surgical intervention. However, the presence of a DU represents an important risk factor for a poor outcome requiring careful planning and resource allocation to provide optimal outcomes. Finally, DU in the vascular surgery population may represent a disproportionate increase in healthcare resource utilization.

\section{Acknowledgments}

National Institutes of Health under Award Number T32HL007849 supported this work. The content is solely the responsibility of the authors and does not necessarily represent the official views of the National Institutes of Health. 


\section{References}

1. Bauer K, Rock K, Nazzal M, Jones O, Qu W. Pressure Ulcers in the United States' Inpatient Population From 2008 to 2012: Results of a Retrospective Nationwide Study. Ostomy Wound Manage. 2016; 62(11):30-8.

2. Allman RM, Goode PS, Burst N, Bartolucci AA, Thomas DR. Pressure ulcers, hospital complications, and disease severity: impact on hospital costs and length of stay. Adv Wound Care. 1999; 12(1):22-30. [PubMed: 10326353]

3. Allman RM, Laprade CA, Noel LB, Walker JM, Moorer CA, Dear MR, et al. Pressure sores among hospitalized patients. Ann Intern Med. 1986; 105(3):337-42. [PubMed: 3740674]

4. Bansal C, Scott R, Stewart D, Cockerell CJ. Decubitus ulcers: a review of the literature. Int J Dermatol. 2005; 44(10):805-10. [PubMed: 16207179]

5. Eachempati SR, Hydo LJ, Barie PS. Factors influencing the development of decubitus ulcers in critically ill surgical patients. Crit Care Med. 2001; 29(9):1678-82. [PubMed: 11546964]

6. Tschannen D, Bates O, Talsma A, Guo Y. Patient-specific and surgical characteristics in the development of pressure ulcers. Am J Crit Care. 2012; 21(2):116-25. [PubMed: 22381988]

7. Frankel H, Sperry J, Kaplan L. Risk factors for pressure ulcer development in a best practice surgical intensive care unit. Am Surg. 2007; 73(12):1215-7. [PubMed: 18186374]

8. Cullum N, Petherick E. Pressure ulcers. BMJ Clin Evid. 2008; 2008

9. Reddy M. Pressure ulcers. BMJ Clin Evid. 2011; 2011

10. Brem H, Nierman DM, Nelson JE. Pressure ulcers in the chronically critically ill patient. Crit Care Clin. 2002; 18(3):683-94. [PubMed: 12140919]

11. Flattau A, Blank AE. Risk factors for 90-day and 180-day mortality in hospitalised patients with pressure ulcers. Int Wound J. 2014; 11(1):14-20. [PubMed: 22738290]

12. Feinglass J, McCarthy WJ, Slavensky R, Manheim LM, Martin GJ. Functional status and walking ability after lower extremity bypass grafting or angioplasty for intermittent claudication: results from a prospective outcomes study. J Vasc Surg. 2000; 31(1 Pt 1):93-103. [PubMed: 10642712]

13. Vermillion C. Operating room acquired pressure ulcers. Decubitus. 1990; 3(1):26-30.

14. Corniello AL, Moyse T, Bates J, Karafa M, Hollis C, Albert NM. Predictors of pressure ulcer development in patients with vascular disease. J Vasc Nurs. 2014; 32(2):55-62. [PubMed: 24944172]

15. Gutacker N, Bloor K, Cookson R. Comparing the performance of the Charlson/Deyo and Elixhauser comorbidity measures across five European countries and three conditions. Eur J Public Health. 2015; 25(Suppl 1):15-20.

16. LaPar DJ, Bhamidipati CM, Harris DA, Kozower BD, Jones DR, Kron IL, et al. Gender, race, and socioeconomic status affects outcomes after lung cancer resections in the United States. Ann Thorac Surg. 2011; 92(2):434-9. [PubMed: 21704976]

17. LaPar DJ, Bhamidipati CM, Mery CM, Stukenborg GJ, Jones DR, Schirmer BD, et al. Primary payer status affects mortality for major surgical operations. Ann Surg. 2010; 252(3):544-50. discussion 50-1. [PubMed: 20647910]

18. LaPar DJ, Nagji AS, Bhamidipati CM, Kozower BD, Lau CL, Ailawadi G, et al. Seasonal variation influences outcomes following lung cancer resections. Eur J Cardiothorac Surg. 2011; 40(1):8390. [PubMed: 21169031]

19. LaPar DJ, Stukenborg GJ, Guyer RA, Stone ML, Bhamidipati CM, Lau CL, et al. Primary payer status is associated with mortality and resource utilization for coronary artery bypass grafting. Circulation. 2012; 126(11 Suppl 1):S132-9. [PubMed: 22965973]

20. Thomas DR, Goode PS, Tarquine PH, Allman RM. Hospital-acquired pressure ulcers and risk of death. J Am Geriatr Soc. 1996; 44(12):1435-40. [PubMed: 8951312] 


\section{Table 1}

Patient demographics and major procedure by presence/absence of ulcer.

\begin{tabular}{|c|c|c|c|}
\hline & No Ulcer $\mathrm{N}=\mathbf{5 2 2 , 9 3 0}$ & Ulcer $N=15,877$ & p-value \\
\hline Carotid Endarterectomy Open & $115,746(22.1)$ & $261(1.6)$ & $<0.001$ \\
\hline Carotid Artery Stent & $40,681(7.8)$ & $261(1.6)$ & $<0.001$ \\
\hline Abdominal Aneurysm Open & $37,747(7.2)$ & $463(2.9)$ & $<0.001$ \\
\hline Abdominal Aneurysm Stent & $38,287(7.3)$ & $187(1.2)$ & $<0.001$ \\
\hline Femoral-Distal Open & $100,573(19.2)$ & $2,519(15.9)$ & $<0.001$ \\
\hline Femoral-Distal Stent & $29,843(5.7)$ & $415(2.6)$ & $<0.001$ \\
\hline Amputation (above/below knee) & $57,457(11.0)$ & $8,508(53.6)$ & $<0.001$ \\
\hline Peripheral Artery Stent & $156,925(30.0)$ & $4,974(31.3)$ & $<0.001$ \\
\hline Age (years) & $66.5 \pm 0.02$ & $70.1 \pm 0.11$ & $<0.001$ \\
\hline Gender (male) & $304,599(58.3)$ & $8,604(54.2)$ & $<0.001$ \\
\hline \multicolumn{4}{|l|}{ Race } \\
\hline White & $334,001(75.5)$ & $8,111(60.2)$ & \multirow{3}{*}{$<0.001$} \\
\hline Black & $54,251(12.3)$ & $3,348(24.8)$ & \\
\hline Hispanic & $32,277(7.3)$ & $1,418(10.5)$ & \\
\hline Alcohol Abuse & $12,906(2.5)$ & $319(2.0)$ & $<0.001$ \\
\hline Deficiency Anemia & $90,125(17.2)$ & $5,779(36.4)$ & $<0.001$ \\
\hline Congestive Heart Failure & $42,587(8.1)$ & $3,715(23.4)$ & $<0.001$ \\
\hline COPD & $113,957(21.8)$ & $3,035(19.1)$ & $<0.001$ \\
\hline Coagulopathy & $23,639(4.5)$ & $1,124(7.1)$ & $<0.001$ \\
\hline Diabetes (uncomplicated) & $122,838(23.5)$ & $3,106(19.6)$ & $<0.001$ \\
\hline Diabetes (chronic complications) & $53,421(10.2)$ & $4,378(27.6)$ & $<0.001$ \\
\hline Drug Abuse & $5,613(1.1)$ & $202(1.3)$ & 0.009 \\
\hline Hypertension & $373,878(71.5)$ & $10,462(65.0)$ & $<0.001$ \\
\hline Liver Disease & $8,038(1.5)$ & $356(2.2)$ & $<0.001$ \\
\hline Fluid/Electrolyte Disorders & $84,522(16.2)$ & $5,710(36.0)$ & $<0.001$ \\
\hline Other Neurological Disorders & $18,877(3.6)$ & $1,670(10.5)$ & $<0.001$ \\
\hline Obesity & $39,840(7.6)$ & $1,251(7.9)$ & 0.12 \\
\hline Paralysis & $11,702(2.2)$ & 1,847 (11.6) & $<0.001$ \\
\hline Peripheral Vascular Disease & $193,984(37.1)$ & $7,835(49.3)$ & $<0.001$ \\
\hline Renal Failure & $106,614(20.4)$ & $6,096(38.4)$ & $<0.001$ \\
\hline Valvular Disorder & $18,930(3.6)$ & $767(4.8)$ & $<0.001$ \\
\hline
\end{tabular}

$\mathrm{N}(\%)$ or mean \pm standard deviation; Chi-squared test or independent samples t-test. COPD: Chronic Obstructive Pulmonary Disease. 


\section{Table 2}

Administrative data by presence or absence of ulcer.

\begin{tabular}{lccc}
\hline & No Ulcer N=522,930 & Ulcer N=15,877 & p-value \\
\hline Non-Elective Admission & $225,804(43.3)$ & $\mathbf{1 1 , 8 0 7}(\mathbf{7 4 . 4})$ & $<0.001$ \\
Admission Day is a Weekend & $43,238(8.3)$ & $\mathbf{2 , 0 6 3}(\mathbf{1 3 . 0})$ & $<0.001$ \\
Transfer in Indicator & & & \\
Not transferred in & $\mathbf{4 8 6 , 5 5 9}(\mathbf{9 3 . 3})$ & $13,134(82.9)$ & \\
Transferred in from a different acute care hospital & $25,678(4.9)$ & $\mathbf{1 , 4 9 0}(\mathbf{9 . 4})$ & $<0.001$ \\
Transferred in from another type of health facility & $9,539(1.8)$ & $\mathbf{1 , 2 1 8}(\mathbf{7 . 7})$ & \\
Primary Expected Payor & & & \\
Medicare & $340,309(65.2)$ & $\mathbf{1 2 , 2 5 2}(\mathbf{7 7 . 3})$ & $<0.001$ \\
Medicaid & $35,490(6.8)$ & $\mathbf{1 , 2 1 5}(\mathbf{7 . 7})$ & \\
Private Insurance & $\mathbf{1 2 0 , 2 2 8}(\mathbf{2 3 . 0})$ & $1,905(12.0)$ & \\
\hline
\end{tabular}

$\mathrm{N}(\%)$; Chi-squared test. 


\section{Table 3}

Patient outcomes by presence or absence of ulcer.

\begin{tabular}{|c|c|c|c|}
\hline & No Ulcer & Ulcer & p-value \\
\hline Died During Admission & $14,322(2.7)$ & $1,004(6.3)$ & $<0.001$ \\
\hline \multicolumn{4}{|l|}{ Complications } \\
\hline Wound & $21,777(4.2)$ & $963(6.1)$ & $<0.001$ \\
\hline Infectious & $5,799(1.1)$ & $376(2.4)$ & $<0.001$ \\
\hline Renal/Urinary & $3,067(0.6)$ & $40(0.3)$ & $<0.001$ \\
\hline Gastrointestinal & $4,276(0.8)$ & $83(0.5)$ & $<0.001$ \\
\hline Systemic & $3,697(0.7)$ & $123(0.8)$ & 0.71 \\
\hline Procedural & $13,507(2.6)$ & $458(2.9)$ & 0.01 \\
\hline Cardiovascular & $21,266(4.1)$ & $721(4.5)$ & 0.002 \\
\hline Neurologic/Stroke & $3,313(0.6)$ & $57(0.4)$ & $<0.001$ \\
\hline Pulmonary & $41,769(8.0)$ & 2,892 (18.2) & $<0.001$ \\
\hline \multicolumn{4}{|l|}{ Total Complications } \\
\hline 0 & $427,295(81.7)$ & $11,199(70.5)$ & \multirow{2}{*}{$<0.001$} \\
\hline 1 & $76,859(14.7)$ & $3,807(24.0)$ & \\
\hline 2 or more & $18,777(3.6)$ & $871(5.5)$ & \\
\hline Failure To Rescue & $7,950(1.5)$ & $503(3.2)$ & $<0.001$ \\
\hline Length of Stay (days) & $6.6 \pm 0.13$ & $17.0 \pm 0.14$ & $<0.001$ \\
\hline \multicolumn{4}{|l|}{ Disposition } \\
\hline Home & $335,560(64.2)$ & $1,630(10.3)$ & \\
\hline Home Health Care & $66,633(12.7)$ & $2,634(16.6)$ & $<0.001$ \\
\hline Another Health Care Facility & $99,205(19.0)$ & $10,259(64.6)$ & \\
\hline Short-Term Hospital & $5,604(1.1)$ & 300 (1.9) & \\
\hline
\end{tabular}

$\mathrm{N}(\%)$ or mean \pm standard deviation; Chi-squared test or independent samples t-test. 


\section{을 \\ }

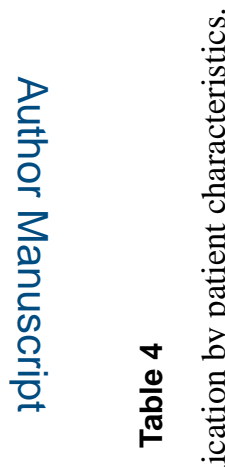

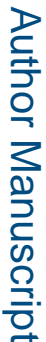

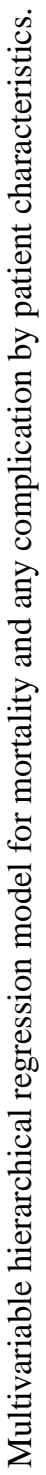

|气

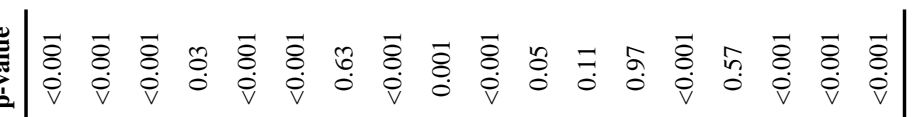

ㅇำ

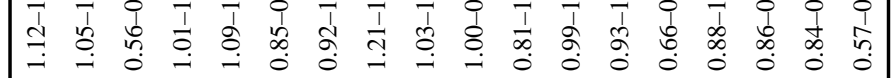

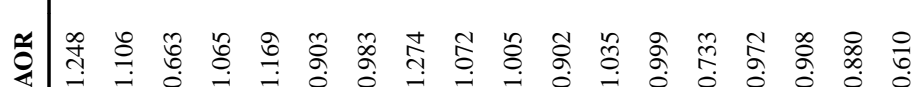

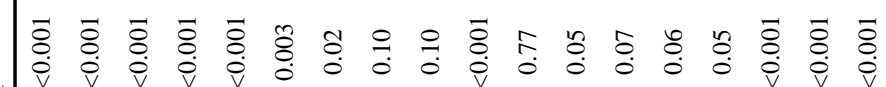
ป

오. 


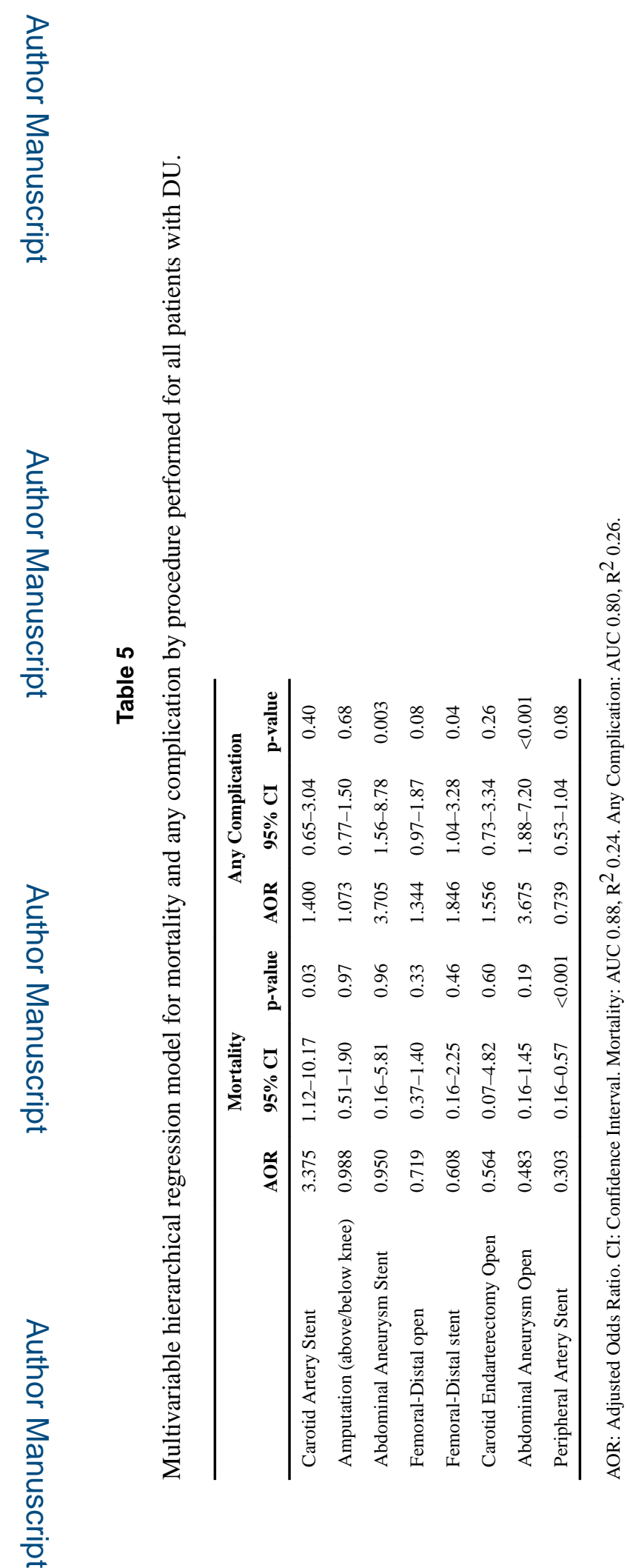

Surgery. Author manuscript; available in PMC 2018 June 01. 


\section{Table 6}

Adjusted odds ratios for discharge disposition.

\begin{tabular}{lc}
\hline & AOR (95\% CI) \\
\hline Risk of having an ulcer associated with discharge to skilled nursing facility & $2.99(2.66-3.37)$ \\
$\begin{array}{l}\text { Admission from non-health care center for elective surgery (known ulcer on admission) associated with discharge to } \\
\text { skilled nursing facility }\end{array}$ & $12.04(7.12-30.35)$ \\
\hline
\end{tabular}

AOR, Adjusted Odds Ratio; CI, Confidence Interval

Reference, Home \pm services 